\title{
Antioxidant effects of Cirsium setidens extract on oxidative stress in human mesenchymal stem cells
}

\author{
JUN HEE LEE ${ }^{1,2}$, HO KYUNG JUNG ${ }^{3}$, YONG-SEOK HAN ${ }^{4}$, YEO MIN YOON ${ }^{4}$, \\ CHUL WON YUN ${ }^{4}$, HWA YEON SUN ${ }^{5}$, HYUN WOO CHO ${ }^{2}$ and SANG HUN LEE ${ }^{4}$
}

\begin{abstract}
${ }^{1}$ Department of Physiology, Laboratory for Vascular Medicine and Stem Cell Biology, Medical Research Institute, School of Medicine, Pusan National University, Yangsan, South Gyeongsang 50612, Republic of Korea;

${ }^{2}$ Department of Neurology, Neuroregeneration and Stem Cell Programs, Institute for Cell Engineering,

The Johns Hopkins University School of Medicine, Baltimore, MD 21287, USA;

${ }^{3}$ Department of Herb Research, Jeollanamdo Development Institute of Traditional Korean Medicine, Jangheung, South Jeolla 59338; ${ }^{4}$ Department of Biochemistry, Medical Science Research Institute, Soonchunhyang University College of Medicine, Cheonan, South Chungcheong 31151; ${ }^{5}$ Department of Urology, Soonchunhyang University Hospital, Soonchunhyang University College of Medicine, Seoul 04401, Republic of Korea
\end{abstract}

Received March 2, 2016; Accepted August 15, 2016

DOI: $10.3892 / \mathrm{mmr} .2016 .5706$

\begin{abstract}
Human mesenchymal stem cells (MSCs) may be used in cell-based therapy to promote neovascularization for the treatment of ischemic diseases. However, high levels of reactive oxygen species (ROS) derived from the pathophysiological ischemic environment induce senescence and apoptosis of MSCs, resulting in reduced functionality and defective neovascularization. Therefore, the present study aimed to determine the protective effects of Cirsium setidens, a natural product, on oxidative stress-induced apoptosis in MSCs. The present study investigated for the change of ROS levels in MSCs using ROS assays. In addition, cell viability determined by MTT and TUNEL assays. Western blot analysis was performed to investigate the change of apoptosis-associated proteins in MSCs. Treatment of MSCs with hydrogen peroxide $\left(\mathrm{H}_{2} \mathrm{O}_{2} ; 200 \mu \mathrm{M}\right)$ significantly increased intracellular ROS levels and cell death; however, pretreatment with $C$. setidens (100 $\mu \mathrm{g} / \mathrm{ml}$ ) suppressed $\mathrm{H}_{2} \mathrm{O}_{2}$-induced ROS generation and increased the survival of MSCs. $\mathrm{H}_{2} \mathrm{O}_{2}$-induced ROS production increased the levels of phosphorylated-p38 mitogen activated protein kinase, c-Jun N-terminal kinase, ataxia telangiectasia mutated and p53; these increases were inhibited by pretreatment with $C$. setidens. In addition, $C$. setidens
\end{abstract}

Correspondence to: Dr Sang Hun Lee, Department of Biochemistry, Medical Science Research Institute, Soonchunhyang University College of Medicine, 44 Suncheonhyang 4-gil, Cheonan, South Chungcheong 31151, Republic of Korea

E-mail: ykckss1114@nate.com; jhlee0407@sch.ac.kr

Key words: Cirsium setidens, mesenchymal stem cells, oxidative stress, apoptosis, antioxidant inhibited ROS-induced apoptosis of MSCs by increasing the expression levels of the anti-apoptotic protein B-cell lymphoma 2 (BCL-2), and decreasing the expression levels of the proapoptotic protein BCL-2-associated X protein. These findings indicated that pretreatment of MSCs with C. setidens may prevent ROS-induced oxidative injury by regulating the oxidative stress-associated signaling pathway, and suppressing the apoptosis-associated signal pathway. Therefore, C. setidens may be developed as a beneficial broad-spectrum agent for enhancing the effectiveness of MSC transplantation in the treatment of ischemic diseases.

\section{Introduction}

Mesenchymal stem cells (MSCs) are a promising cell source for regenerative medicine due to their potential for self-renewal and multilineage differentiation into bone, cartilage, muscle, ligament, tendon, adipose, and endothelial cells (1). The isolation of MSCs from human donors or patients is relatively easy, and MSC cultures are able to expand rapidly for $\geq 30$ population doublings. In addition, the differentiation of MSCs into various phenotypes is simple in lineage-specific culture conditions. These properties make MSCs attractive candidates for the treatment of various diseases, and $>500$ clinical trials are being conducted using MSCs to overcome a diverse range of diseases (2).

Despite their beneficial effects, the application of MSCs is limited due to pathophysiological environmental conditions, including oxidative stress, inflammation, low oxygen levels and restricted nutrient supply (3). Various stress conditions trigger reduced proliferation and loss of stemness, and are able to induce senescence, resulting in $>99 \%$ cell death during the first few days following MSC transplantation (4-7). Therefore, protection against several stressors and optimization of MSC culture conditions are required to produce functional MSCs with high therapeutic efficiency. To address this issue, preconditioning, 
hypoxic culture, pretreatment and genetic manipulation have been suggested to increase the survival of MSCs (3).

Cirsium setidens is a wild perennial herb that possesses various bioactivities, including antitumor, antioxidant and hepatoprotective effects (8-10). C. setidens, which is a bioactive flavonoid, has previously been used to treat hemostasis, hematemesis, hematuria and hypertension (11). Although C. setidens exerts various biological activities, there is no evidence regarding the protective effects of $C$. setidens against oxidative stress in MSCs. The present study aimed to assess the effects of $C$. setidens on ROS-induced oxidative stress in MSCs, and to elucidate the mechanism underlying its anti-apoptotic effects against oxidative stress.

\section{Materials and methods}

MSCs culture conditions. Human adipose tissue-derived MSCs were obtained from the American Type Culture Collection (Manassas, VA, USA), and were confirmed to be pathogenand mycoplasma-free. The supplier certified that the MSCs expressed specific cell surface markers [cluster of differentiation (CD)73 and CD105, but not CD31], and had adipogenic and osteogenic differentiation potential when cultured with specific differentiation media. MSCs were cultured in $\alpha$-minimum essential medium ( $\alpha$-MEM; Gibco; Thermo Fisher Scientific, Inc., Waltham, MA, USA) supplemented with $10 \%$ (v/v) fetal bovine serum (FBS; Gibco; Thermo Fisher Scientific, Inc.), $100 \mathrm{U} / \mathrm{ml}$ penicillin and $100 \mu \mathrm{g} / \mathrm{ml}$ streptomycin. MSC cultures were grown in a $5 \% \mathrm{CO}_{2}$ humidified incubator at $37^{\circ} \mathrm{C}$.

Preparation of $C$. setidens water extract. C. setidens was collected from the Jeongseon-Gondre Farming Association Corporation (Jeongseon-gun, South Korea). C. setidens was extracted by suspending $100 \mathrm{~g}$ into $5 \mathrm{~L}$ of hot distilled water for $3 \mathrm{~h}$. The water extract was filtered to remove plant particles through filter paper and was then concentrated in a vacuum under reduced pressure and lyophilized using a freeze dryer for three days in order to fully dry the sample. In total, $19.2 \mathrm{~g}$ C. setidens extracted powder was recovered and was maintained at $-20^{\circ} \mathrm{C}$. The powder was then dissolved in phosphate buffer $(\mathrm{pH} 7.4,20 \mathrm{mg} / \mathrm{ml})$ and was stored at $-80^{\circ} \mathrm{C}$.

High-performance liquid chromatography (HPLC). A Shimadzu LC-20A (Shimadzu Corporation, Kyoto, Japan), consisting of two LC-20 AD Pumps, a DGU-20A3 vacuum degasser, a SPD-M20A photodiode array detector (PDA) and a CTO 20A Autosampler, was employed. The chromatographic analysis for determination of pectolinarin was carried out using a Luna 5 u C18 100A column (250x4.6 mm; Phenomenex, Inc., Torrance, CA, USA). The mobile phase consisted of solvent A $(0.1 \%$ formic acid in water) and $100 \%$ acetonitrile (solvent B). The solvent gradient elution conditions were $5 \%$ (B) for $0-5 \mathrm{~min}$, $5-60 \%$ (B) for 5-22 $\mathrm{min}, 60-60 \%$ (B) for $22-24 \mathrm{~min}$ and $60-5 \%$ (B) for $24-30 \mathrm{~min}$ at a flow-rate of $1.0 \mathrm{ml} / \mathrm{min}$, and the column oven was operated at $40^{\circ} \mathrm{C}$ throughout the study. Detection was conducted with different wavelengths of $273 \mathrm{~nm}$. Injection volume of the sample solutions was $10 \mu 1$. Peak identity was confirmed by comparison of spectra obtained from the PDA detector. A spectrum of pectolinarin (Sigma-Aldrich; Merck Millipore, Darmstadt, Germany) and $C$. setidens water extract were compared and analyzed using PDA, at a wavelength of 200-400 $\mathrm{nm}$. The data of chromatogram and spectrum were stored and displayed on a computer.

Chemical treatment of MSCs. MSCs were washed twice with phosphate-buffered saline, and the medium was replaced with fresh $\alpha$-MEM supplemented with $10 \%$ FBS. To assess cell viability, MSCs were pretreated with $C$. setidens $(100 \mu \mathrm{g} / \mathrm{ml}$ or $200 \mu \mathrm{g} / \mathrm{ml}$ ) at $37^{\circ} \mathrm{C}$ for $30 \mathrm{~min}$, and were then treated with hydrogen peroxide $\left(\mathrm{H}_{2} \mathrm{O}_{2} ; 200 \mu \mathrm{M}\right)$ for the indicated durations $(0,4,6$ and $8 \mathrm{~h})$. To investigate various cell signaling pathways, MSCs were treated with $\mathrm{H}_{2} \mathrm{O}_{2}(200 \mu \mathrm{M})$ for the indicated duration $(0,15,30,60$ and $120 \mathrm{~min})$. MSCs were treated with C. setidens $(100 \mu \mathrm{g} / \mathrm{ml})$ for $30 \mathrm{~min}$ and were then treated with $\mathrm{H}_{2} \mathrm{O}_{2}(200 \mu \mathrm{M})$ for $120 \mathrm{~min}$.

Cell viability assay. Subconfluent, exponentially growing MSCs were incubated in a 96-well plate with $C$. setidens for various durations. Cell viability was determined using a modified 3-(4,5-dimethyl-thiazol-2-yl)-2,5-diphenyltetrazolium bromide assay, which is based on the conversion of the tetrazolium salt 3-(4,5-dimethylthiazol-2-yl)-5-(3-carboxymethoxyphenyl)-2 -(4-sulfophenyl)-2-tetrazolium to formazan by mitochondrial $\mathrm{NAD}(\mathrm{P}) \mathrm{H}$-dependent oxidoreductase enzymes. After $4 \mathrm{~h}$ formazan levels were quantified by measuring the absorbance at $575 \mathrm{~nm}$ using a microplate reader (Tecan Group AG, Männedorf, Switzerland).

Intracellular reactive oxygen species (ROS) assay. CM-H2DCFDA (DCF-DA), which acts as $\mathrm{H}_{2} \mathrm{O}_{2}$-sensitive fluorophore, was used to detect the $\mathrm{H}_{2} \mathrm{O}_{2}$-induced production of ROS. DCF-DA $(10 \mu \mathrm{M})$ was added to the cells and incubated for $30 \mathrm{~min}$ at room temperature in the dark. The cells were subsequently observed under a laser confocal microscope (magnification, x600; Fluoview 1000; Olympus Corporation, Tokyo, Japan) with excitation and emission wavelengths of 488 and 515-540 nm, respectively.

Western blot analysis. Total protein was extracted using RIPA Lysis Buffer (Thermo Fisher Scientific, Inc.). Bicinchoninic acid assay kit (Thermo Fisher Scientific, Inc.) was used to quantify proteins. The cell lysate ( $50 \mu \mathrm{g}$ protein) was separated by $10 \%$ sodium dodecyl sulfate-polyacrylamide gel electrophoresis, and the proteins were transferred to nitrocellulose membranes. The membranes were then blocked with $5 \%$ skim milk for $1 \mathrm{~h}$ at room temperature, and were incubated with the primary antibodies at the dilutions recommended by the supplier. Ataxia telangiectasia mutated (ATM; cat. no. sc-377293), phosphorylated (p)-ATM (cat. no. sc-47739), p38 (cat. no. sc-81621), p-p38 (cat. no. sc-101758), c-Jun N-terminal kinase (JNK; cat. no. sc-7345), p-JNK (cat. no. sc-6254), p53(cat. no. sc-126) and p-p53 (cat. no. sc-101762), B-cell lymphoma 2 (BCL-2;cat. no. sc-7382), BCL-2-associated X protein (BAX; cat. no. sc-6236), cleaved poly (ADP ribose) polymerase-1 (PARP-1; cat. no. sc-56196), cleaved caspase-3 (cat. no. sc-7272) and $\beta$-actin (cat. no. sc-47778) primary antibodies were obtained from Santa Cruz Biotechnology, Inc. (Dallas, TX, USA). All primary antibodies were diluted 1:1,000 in 5\% skim milk and incubated with membranes overnight at $4^{\circ} \mathrm{C}$, the membranes were washed, and the primary antibodies were detected 
A

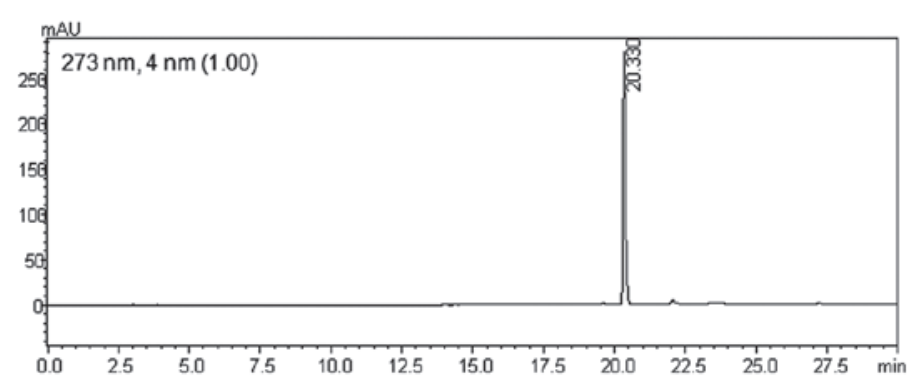

B

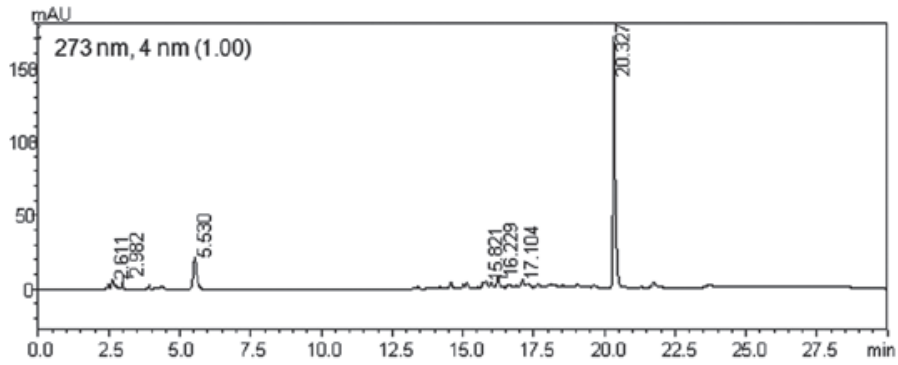

C

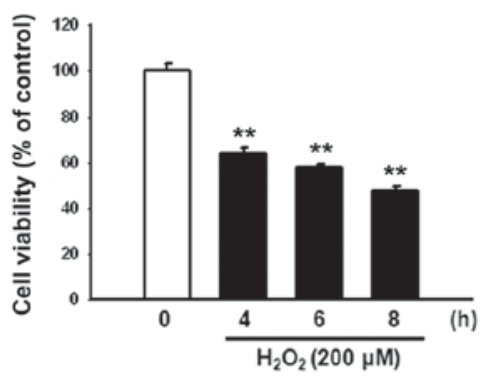

D

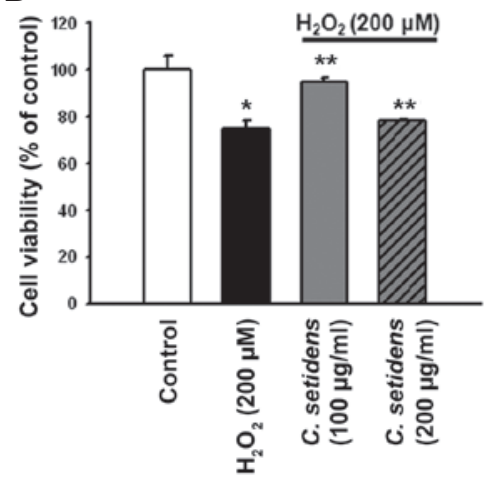

Figure 1. Effects of Cirsium setidens on hydrogen peroxide $\left(\mathrm{H}_{2} \mathrm{O}_{2}\right)$-induced apoptotic cell death of mesenchymal stem cells (MSCs). (A and B) High-performance liquid chromatography (HPLC) chromatograms of (A) pectolinarin and (B) water extract of $C$. setidens. (C) $\mathrm{MSCs}$ were treated with $\mathrm{H}_{2} \mathrm{O}_{2}$ (200 $\mu \mathrm{M}$ ) for $0-8 \mathrm{~h}$, and cell viability was subsequently assessed. (D) Following pretreatment with $C$. setidens (100 and $200 \mu \mathrm{g} / \mathrm{ml})$ for $30 \mathrm{~min}$, cell viability was investigated following $8 \mathrm{~h}$ of exposure to $\mathrm{H}_{2} \mathrm{O}_{2}$. Data are presented as the mean \pm standard error of the mean. ${ }^{*} \mathrm{P}<0.05$ and ${ }^{* * *} \mathrm{P}<0.01$ vs. control (untreated).

following incubation with secondary antibodies horseradish peroxidase-conjugated goat anti-rabbit immunoglobulin $\mathrm{G}$ (IgG; cat. no. sc-2004; 1:10,000) or goat anti-mouse IgG (cat. no. sc-2005; 1:10,000; Santa Cruz Biotechnology, Inc.) for $1 \mathrm{~h}$ at $37^{\circ} \mathrm{C}$. The bands were visualized using enhanced chemiluminescence reagents (Amersham; GE Healthcare Life Sciences, Little Chalfont, UK). The semi-quantification of western blotting bands were used Image J version 1.47 (National Institutes of Health, Bethesda, MD, USA) and compared to $\beta$-actin.

Terminal deoxynucleotidyl transferase (TdT)-mediated dUTP nick end labeling (TUNEL) assay. A TUNEL assay was performed using the TdT Fluorescein In Situ Apoptosis Detection kit (Trevigen, Gaithersburg, MD, USA). The MSCs were pretreated with $C$. setidens for $30 \mathrm{~min}$, and were then treated with $\mathrm{H}_{2} \mathrm{O}_{2}$ for $8 \mathrm{~h}$. MSCs were labeled according to the manufacturer's instructions. Stained MSCs were visualized under a fluorescent microscope (Carl Zeiss, Oberkochen, Germany).
Statistical analysis. All data are presented as the mean \pm standard error of the mean. All experiments were repeated 5 times and were analyzed by one-way analysis of variance, followed by a comparison of the treatment and control groups using the Bonferroni-Dunn test using SPSS version 19 (IBM SPSS, Armonk, NY, USA). $\mathrm{P}<0.05$ was considered to indicate a statistically significant difference.

\section{Results}

C. setidens exerts protective effects against $\mathrm{H}_{2} \mathrm{O}_{2}$-induced cell death in MSCs. Examination of the HPLC chromatograms of $C$. setidens indicated that the extract contained pectolinarin (Fig. 1A and B). To explore the protective effects of $C$. setidens against $\mathrm{H}_{2} \mathrm{O}_{2}$-induced oxidative stress, MSCs were treated with $\mathrm{H}_{2} \mathrm{O}_{2}(200 \mu \mathrm{M})$ for the indicated time periods $(0,4,6$ and $8 \mathrm{~h})$. The maximal effect of $\mathrm{H}_{2} \mathrm{O}_{2}$ on MSC cell death was observed following $8 \mathrm{~h}$ of treatment (Fig. 1C). MSCs were pretreated with $C$. setidens $(0,100$ or $200 \mu \mathrm{g} / \mathrm{ml})$ for $30 \mathrm{~min}$ and were then treated with $\mathrm{H}_{2} \mathrm{O}_{2}$ for $8 \mathrm{~h}$. Pretreatment with $C$. setidens 

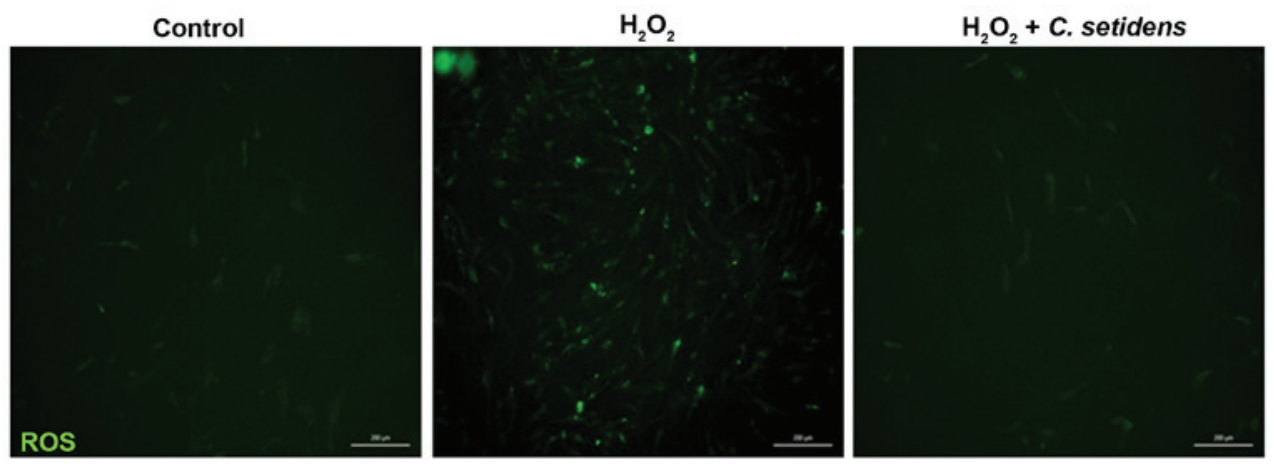

Figure 2. Cirsium setidens inhibits intracellular reactive oxygen species (ROS) generation. Mesenchymal stem cells were treated with $C$. setidens (100 $\mu \mathrm{g} / \mathrm{ml})$ for 30 min prior to hydrogen peroxide $\left(\mathrm{H}_{2} \mathrm{O}_{2} ; 200 \mu \mathrm{M}\right)$ treatment; cellular ROS levels were assessed using the ROS-sensitive fluorophore CM-H2DCFDA (DCF-DA). Images were captured under a confocal microscope and are representative of four independent experiments. Scale bar, $200 \mu \mathrm{m}$.

A

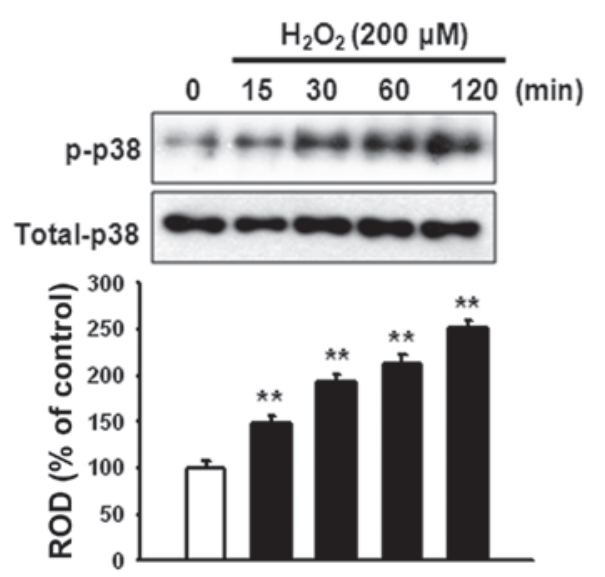

C
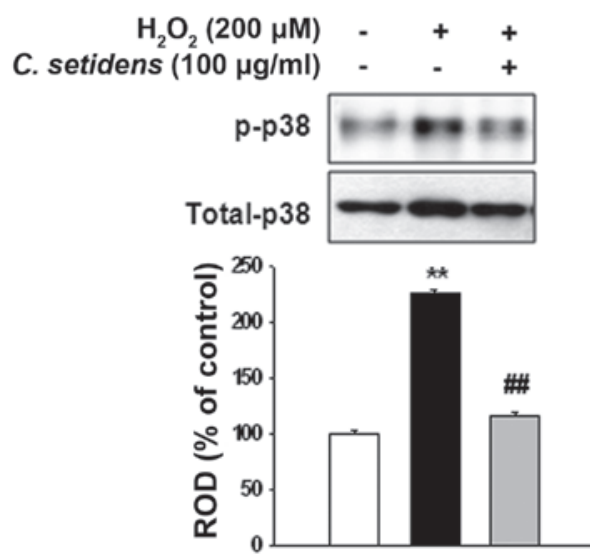

B
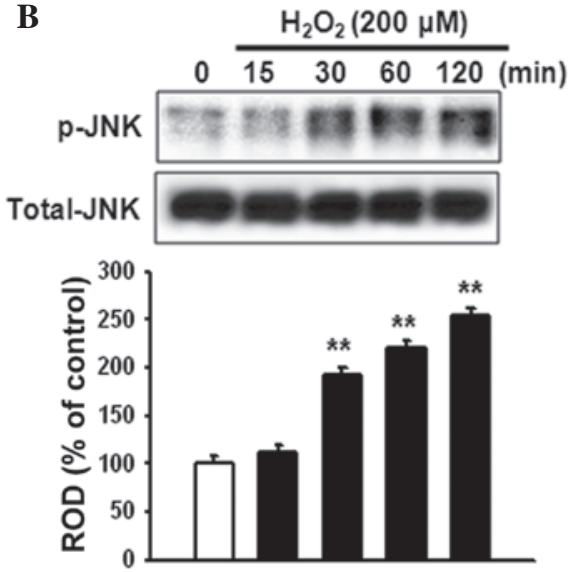

D
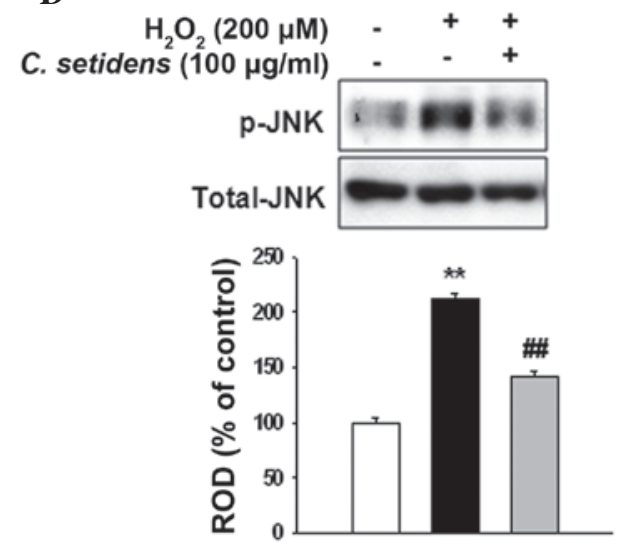

Figure 3. Cirsium setidens inhibits the activation of p38 mitogen-activated protein kinase (MAPK) and c-Jun N-terminal kinase (JNK). (A and B) Mesenchymal stem cells (MSCs) were treated with hydrogen peroxide $\left(\mathrm{H}_{2} \mathrm{O}_{2} ; 200 \mu \mathrm{M}\right)$ for 0-120 min, and the levels of phosphorylated (p)-p38 MAPK and p-JNK were assessed by western blot analysis. Bar graphs represent the relative expression levels of p-p38 and p-JNK normalized to total p38 and total JNK, respectively. (C and D) MSCs were treated with C. setidens $\left(100 \mu \mathrm{g} / \mathrm{ml}\right.$ ), followed by $\mathrm{H}_{2} \mathrm{O}_{2}$, and the levels of p-p38 MAPK and p-JNK were assessed by western blot analysis. Bar graphs represent the relative expression levels of p-p38 and p-JNK normalized to total p38 and total JNK, respectively. Data are presented as the mean \pm standard error of the mean. ${ }^{* *} \mathrm{P}<0.01$ vs. control (untreated); ${ }^{\# \#} \mathrm{P}<0.01$ vs. treatment with $\mathrm{H}_{2} \mathrm{O}_{2}$ alone. ROD, relative optical density.

significantly increased the viability of MSCs, particularly when used at a concentration of $100 \mu \mathrm{g} / \mathrm{ml}$ (Fig. 1D).

C. setidens mediates the inhibition of $\mathrm{H}_{2} \mathrm{O}_{2}$-induced $\mathrm{ROS}$ generation and phosphorylation of stress-associated mitogen-activated protein kinases (MAPKs). To investigate the inhibitory effects of $C$. setidens on ROS generation in MSCs, DCF-DA was used as an indicator of ROS production, and alterations to intracellular peroxide levels were assessed. Following treatment of MSCs with $\mathrm{H}_{2} \mathrm{O}_{2}(200 \mu \mathrm{M})$, the intracellular ROS levels were markedly increased compared with in the untreated cells. Conversely, pretreatment of MSCs with 
A

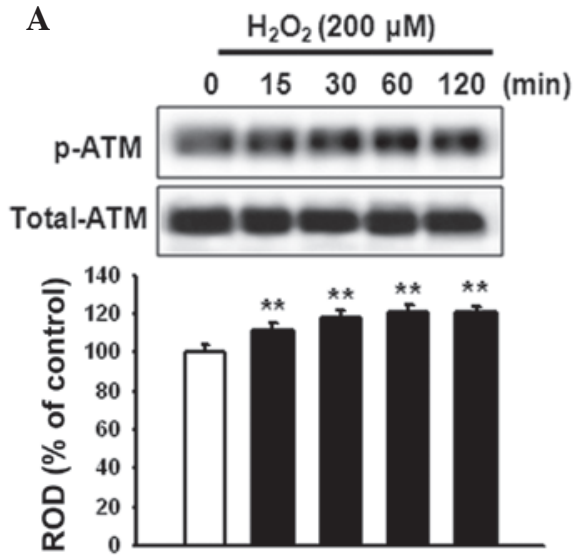

C

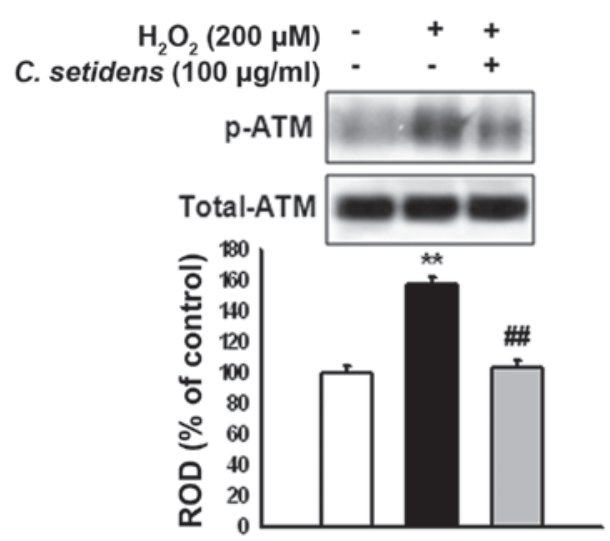

B
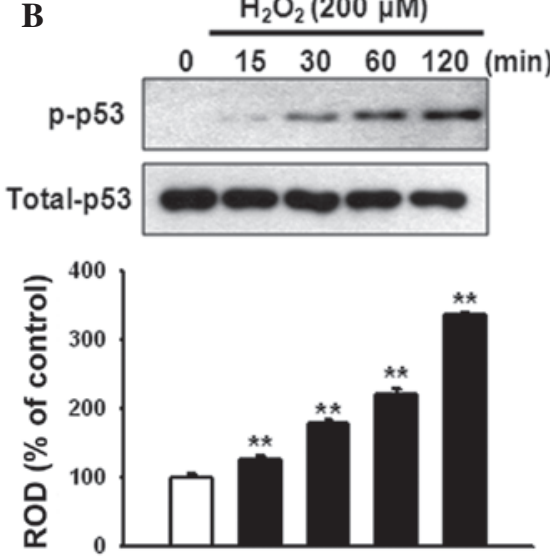

D

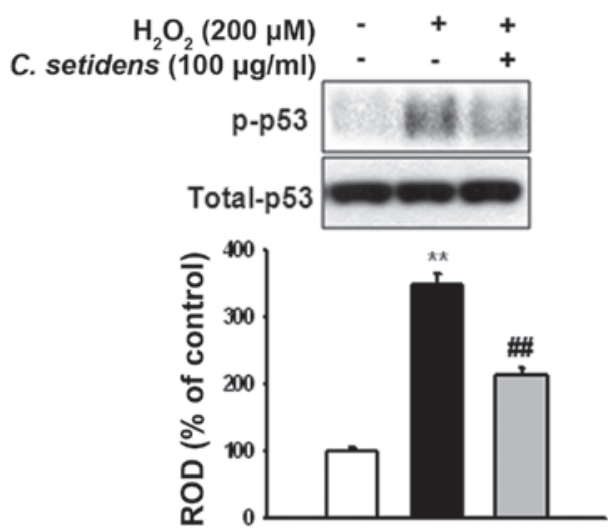

Figure 4. Cirsium setidens inhibits the ataxia telangiectasia mutated (ATM) and p53 pathways. (A and B) Mesenchymal stem cells (MSCs) were treated with hydrogen peroxide $\left(\mathrm{H}_{2} \mathrm{O}_{2} ; 200 \mu \mathrm{M}\right)$ for 0-120 min, and the levels of phosphorylated (p)-ATM and p-p53 were assessed by western blot analysis. Bar graphs represent the relative expression levels of p-ATM and p-p53 normalized to total ATM and total p53, respectively. (C and D) MSCs were treated with C. setidens $(100 \mu \mathrm{g} / \mathrm{ml})$, followed by $\mathrm{H}_{2} \mathrm{O}_{2}$, and the levels of p-ATM and p-p53 were assessed by western blot analysis. Bar graphs represent the relative expression levels of p-ATM and p-p53 normalized to total ATM and total p53, respectively. Data are presented as the mean \pm standard error of the mean. ${ }^{* *} \mathrm{P}<0.01 \mathrm{vs}$. control (untreated); ${ }^{\# \#<0.01 ~ v s . ~ t r e a t m e n t ~ w i t h ~} \mathrm{H}_{2} \mathrm{O}_{2}$ alone. ROD, relative optical density.

C. setidens $(100 \mu \mathrm{g} / \mathrm{ml})$ markedly suppressed intracellular ROS levels (Fig. 2). To determine the regulation of stress-associated MAPKs in ROS-induced cell death, MSCs were treated with $\mathrm{H}_{2} \mathrm{O}_{2}$ for the indicated time periods $(0,15,30,60$ and $120 \mathrm{~min})$. $\mathrm{H}_{2} \mathrm{O}_{2}$ increased the phosphorylation of p38 and JNK MAPKs in a time-dependent manner (Fig. $3 \mathrm{~A}$ and $\mathrm{B}$ ), whereas the phosphorylation of p38 and JNK was significantly decreased following pretreatment with C. setidens (Fig. 3C and D).

Effects of C. setidens on apoptosis regulation in MSCs. To elucidate the involvement of ATM and p53 activation in ROS-induced apoptosis, MSCs were treated with $\mathrm{H}_{2} \mathrm{O}_{2}$ for the indicated time periods $(0,15,30,60$ and $120 \mathrm{~min}) . \mathrm{H}_{2} \mathrm{O}_{2}$ increased the phosphorylation of ATM and p53 in a time-dependent manner (Fig. 4A and B). However, the activation of ATM and p53 was significantly reduced following pretreatment with $C$. setidens (Fig. 4C and D).

Anti-apoptotic effects of $C$. setidens on the regulation of apoptosis-associated proteins in MSCs. To determine whether pretreatment with $C$. setidens may affect ROS-induced expression of apoptotic proteins in MSCs, MSCs were treated with $\mathrm{H}_{2} \mathrm{O}_{2}$ for the indicated time periods $(0,4,6$ and $8 \mathrm{~h}$ ).
Subsequently, the expression levels of BCL-2 (anti-apoptotic protein) and Bax (proapoptotic protein) were assessed using western blot analysis. $\mathrm{H}_{2} \mathrm{O}_{2}$-induced ROS decreased the expression levels of BCL-2 and increased the expression levels of Bax in a time-dependent manner (Fig. 5A); however, pretreatment with $C$. setidens significantly increased BCL-2 expression and decreased Bax expression (Fig. 5B). To further investigate the regulation of key signaling pathways against oxidative stress, following pretreatment with $C$. setidens the expression levels of proapoptotic regulators, cleaved PARP-1 and caspase-3, were detected by western blot analysis. $\mathrm{H}_{2} \mathrm{O}_{2}$-induced ROS activated cleaved PARP-1 and caspase-3, whereas pretreatment with $C$. setidens significantly inhibited their expression (Fig. 5C and D). In addition, the results of a TUNEL assay indicated that pretreatment with $C$. setidens significantly protected against oxidative stress-induced apoptosis of MSCs (Fig. 6A and B).

\section{Discussion}

For applications such as preclinical and clinical trials, the cell source for stem cell-based therapy should be abundant, accessible, easy to deliver to the injured site, tested and 
A
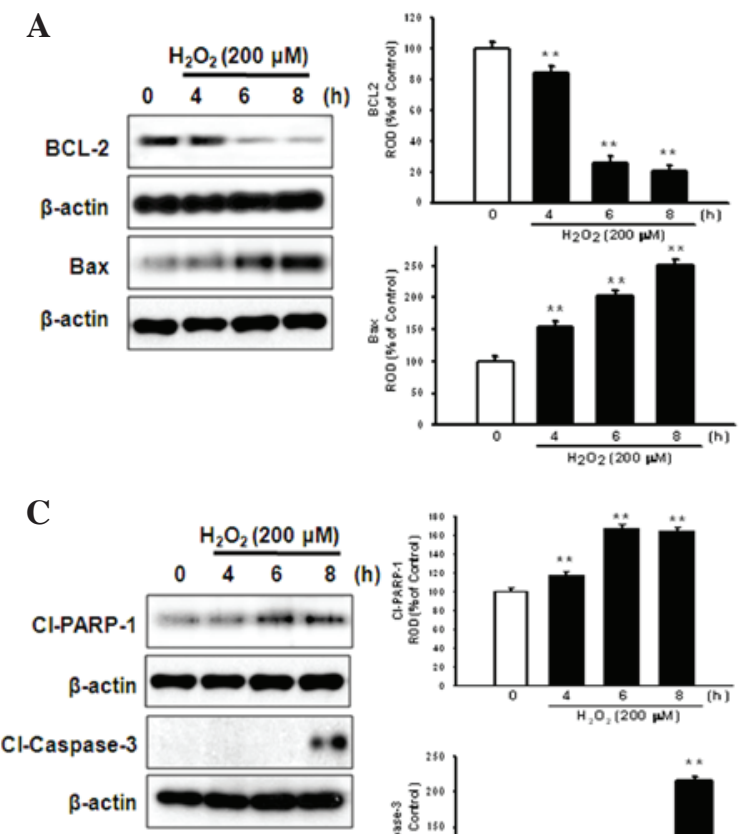
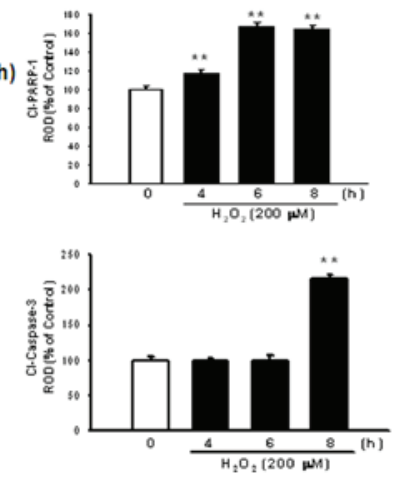

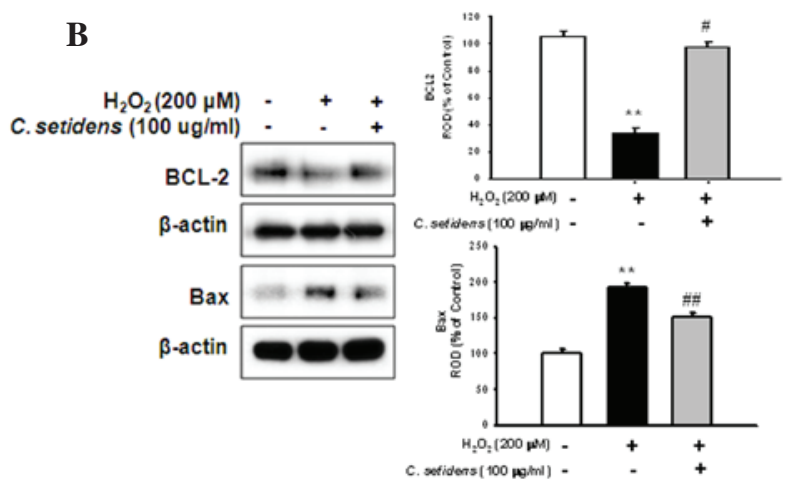

D
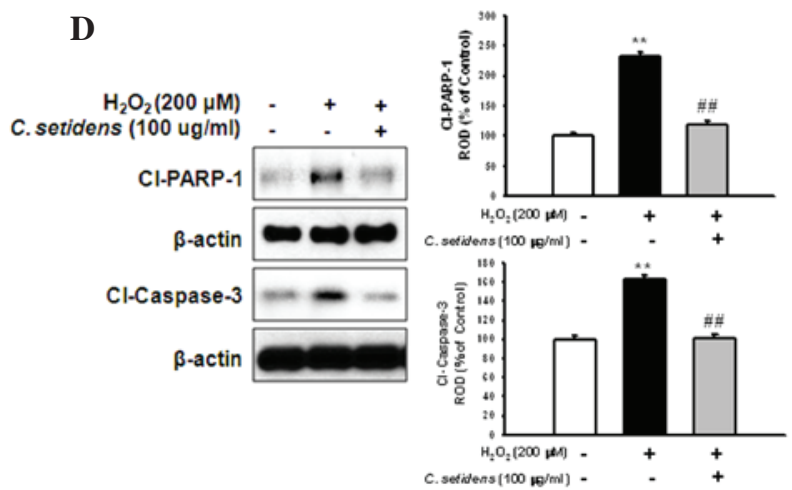

Figure 5. Effects of Cirsium setidens extract on hydrogen peroxide $\left(\mathrm{H}_{2} \mathrm{O}_{2}\right)$-induced regulation of apoptotic proteins in mesenchymal stem cells (MSCs). (A) MSCs were treated with $200 \mu \mathrm{M} \mathrm{H}_{2} \mathrm{O}_{2}$ for 0-8 h, and the expression levels of B-cell lymphoma 2 (BCL-2) and BCL-2-associated X protein (Bax) were detected by western blot analysis. Bar graphs represent the relative expression levels of BCL-2 and Bax normalized to $\beta$-actin. (B) MSCs were pretreated with C. setidens $(100 \mu \mathrm{g} / \mathrm{ml})$ for $30 \mathrm{~min}$, followed by $200 \mu \mathrm{M} \mathrm{H}_{2} \mathrm{O}_{2}(8 \mathrm{~h})$, and the expression levels of BCL-2 and Bax were detected by western blotting. Bar graphs represent the relative expression levels of BCL-2 and Bax normalized to $\beta$-actin. (C) MSCs were treated with $200 \mu \mathrm{M} \mathrm{H}_{2} \mathrm{O}_{2}$ for $0-8 \mathrm{~h}$, and the expression levels of cleaved poly (ADP ribose) polymerase-1 (Cl-PARP-1) and cleaved caspase-3 (Cl-Caspase-3) were detected by western blot analysis. Bar graphs represent the relative expression levels of Cl-PARP-1 and Cl-caspase-3 normalized to $\beta$-actin. (D) MSCs were pretreated with C. setidens $(100 \mu \mathrm{g} / \mathrm{ml})$ for $30 \mathrm{~min}$, followed by $200 \mu \mathrm{M} \mathrm{H}_{2} \mathrm{O}_{2}(8 \mathrm{~h})$, and the expression levels of Cl-PARP-1 and Cl- caspase-3 were detected by western blotting. Bar graphs representsthe relative expression levels of $\mathrm{Cl}$-PARP-1 and Cl-caspase- 3 normalized to $\beta$-actin. Data are presented as the mean \pm standard error of the mean. ${ }^{* *} \mathrm{P}<0.01 \mathrm{vs}$. control (untreated); ${ }^{\#} \mathrm{P}<0.05,{ }^{\# \prime} \mathrm{P}<0.01$ vs. treatment with $\mathrm{H}_{2} \mathrm{O}_{2}$ alone. $\mathrm{ROD}$, relative optical density.

optimized in suitable animal models, and non-tumorigenic and non-immunogenic (12). Among stem cell sources, MSCs exhibit an extensive differentiation potential and are easy to harvest from several tissues, including bone marrow, adipose tissue, and skeletal muscle (1). In particular, adipose-derived MSCs are an attractive cell source due to their abundance, accessibility, multipotency and loss of immunogenicity (13). However, the survival rate and differentiation of transplanted stem cells in injured sites is limited due to apoptosis caused by the pathophysiological environment (3). Therefore, identification of a strategy for the protection of transplanted MSCs is important for the development of MSCs-based therapy for regenerative medicine. C. setidens is a phenolic and flavonoid compound, which possesses anti-inflammatory, anticancer, anti-mutation, antifungal, hepatoprotective and neuroprotective activity, and is capable of immune enhancement $(9,10,14-16)$. In the present study, the HPLC chromatogram detected pectolinarin in the C. setidens extract. Pectolinarin possess anti-inflammatory effects through the inhibition of eicosanoid formation (17). Despite these attractive properties, the protective effects of C. setidens on oxidative injury in MSCs have yet to be fully elucidated.

The present study demonstrated that pretreatment of MSCs with $C$. setidens inhibited $\mathrm{H}_{2} \mathrm{O}_{2}$-induced apoptosis, and reduced ROS generation. Ischemic conditions induce intracellular ROS production, resulting in the activation of inflammation and apoptosis. In addition, oxidative stress activates MAPKs, which contribute to modulation of the stress response (18). MAPK kinase kinases sense the degree of stress-induced cell damage and regulate the MAPK cascade to determine cell fate. Eventually, downstream MAPKs phosphorylate several effectors, including p38 MAPK and JNK, to induce apoptosis (19). $\mathrm{H}_{2} \mathrm{O}_{2}$-induced ROS increased the phosphorylation of p38 and JNK; however, pretreatment of MSCs with C. setidens suppressed the phosphorylation of p38 and JNK. These results suggested that pretreatment with C. setidens may inhibit ROS-induced apoptosis through the regulation of stress-associated MAPKs.

ATM is a pivotal regulatory molecule in ROS-induced apoptosis (20), which has a key role in the cellular response to DNA damage, particularly double strand breaks (21). The phosphorylation of ATM has previously been reported to differ between oxidative stress-induced phosphorylation and double strand break-dependent ATM-mediated phosphorylation (22). Treatment with $\mathrm{H}_{2} \mathrm{O}_{2}$ induces the phosphorylation of ATM (S1981) and its downstream target p53, but not histone 2AX, a double strand break marker, thus suggesting that ROS-induced ATM activation is independent of the double strand break process (23). The results of the present study revealed that 
A

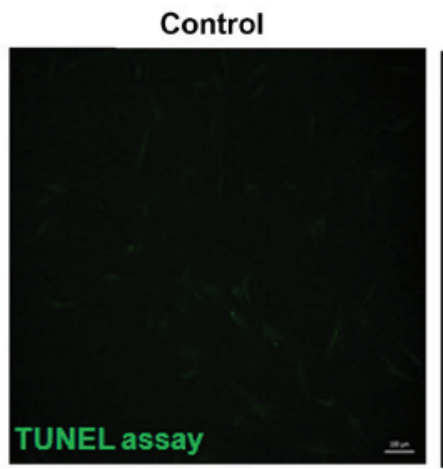

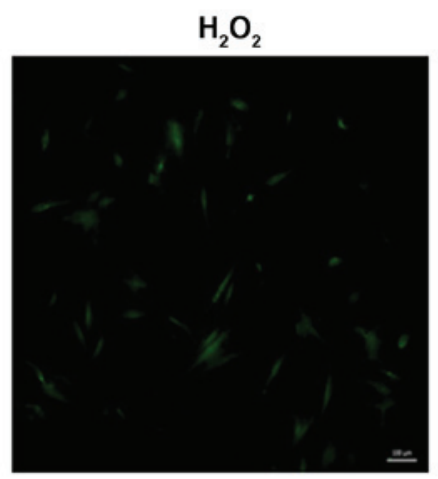

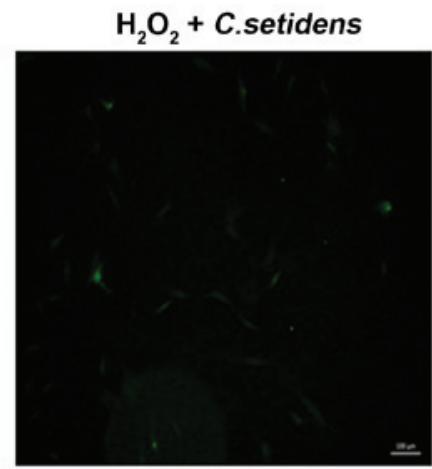

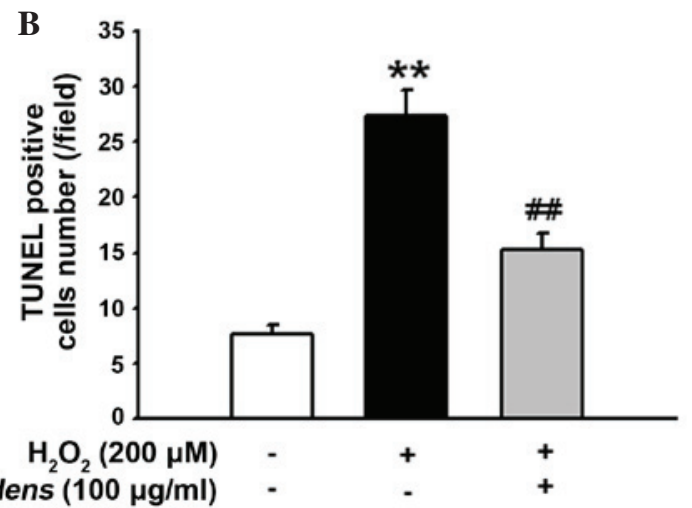

Figure 6. Effects of Cirsium setidens on hydrogen peroxide $\left(\mathrm{H}_{2} \mathrm{O}_{2}\right.$ )-induced apoptosis in mesenchymal stem cells (MSCs). MSCs were pretreated with C. setidens for $30 \mathrm{~min}$, followed by $\mathrm{H}_{2} \mathrm{O}_{2}(200 \mu \mathrm{M})$ for $8 \mathrm{~h}$, and a terminal deoxynucleotidyl transferase-mediated dUTP nick end labeling (TUNEL) assay was conducted. (A) Representative images of TUNEL-positive cells (green). Scale bar, $100 \mu \mathrm{m}$. (B) Bar graph represents the number of TUNEL-positive cells per field. Data are presented as the mean \pm standard error of the mean. ${ }^{* *} \mathrm{P}<0.01$ vs. control; ${ }^{\# \#} \mathrm{P}<0.01$ vs. treatment with $\mathrm{H}_{2} \mathrm{O}_{2}$ alone.

treatment with $C$. setidens decreased the phosphorylation of ATM and p53 in response to oxidative stress, thus indicating that $C$. setidens may reduce oxidative stress-induced cell death via the modulation of ATM and p53 activity, as well as MAPK activation.

Apoptosis occurs under pathophysiological conditions via a cascade of cellular events, which involves various apoptosis-associated genes (24). Pathological apoptosis is induced by downregulation of anti-apoptotic proteins, such as BCL-2, or by the expression of proapoptotic proteins, such as Bax (25). In addition, the cleavage of caspase-3 results in DNA fragmentation, cytoskeletal and nuclear protein degradation, and the expression of ligands for phagocytic cell receptors (25). The cleavage of PARP-1 by caspases is one of the first biochemical markers of apoptosis (26). The present study indicated that $\mathrm{H}_{2} \mathrm{O}_{2}$ induced-ROS decreased the BCL-2/Bax ratio, and increased the cleavage of caspase-3 and PARP-1. Conversely, pretreatment of MSCs with $C$. setidens restored the BCL-2/Bax ratio and reduced activation of caspase-3 and PARP-1. These results suggested that C. setidens may inhibit ROS-induced apoptosis via regulation of the apoptotic cascade.

The present study identified the cytoprotective effects of C. setidens on MSCs exposed to oxidative stress. Under oxidative stress conditions, $C$. setidens protected MSCs from the harmful effects of intracellular ROS via regulation of stress-associated MAPKs, ATM and p53 phosphorylation, and apoptotic signal cascades. These findings suggested that
C. setidens may be developed as a cytoprotective agent for use alongside MSCs-based therapy for the treatment of ischemic diseases.

\section{Acknowledgements}

The present study was supported by the National Research Foundation (NRF) grant funded by the Korean government (MEST) (grant no. 2011-0009610) and the Ministry of Education (grant. no. 2016R1D1A3B01007727). The funders had no role in study design, data collection or analysis, the decision to publish, or preparation of the manuscript.

\section{References}

1. Chamberlain G, Fox J, Ashton B and Middleton J: Concise review: Mesenchymal stem cells: Their phenotype, differentiation capacity, immunological features, and potential for homing. Stem Cells 25: 2739-2749, 2007.

2. Marquez-Curtis LA, Janowska-Wieczorek A, McGann LE and Elliott JA: Mesenchymal stromal cells derived from various tissues: Biological, clinical and cryopreservation aspects. Cryobiology 71: 181-197, 2015.

3. Amiri F, Jahanian-Najafabadi A and Roudkenar MH: In vitro augmentation of mesenchymal stem cells viability in stressful microenvironments: In vitro augmentation of mesenchymal stem cells viability. Cell Stress Chaperones 20: 237-251, 2015.

4. Wei H, Li Z, Hu S, Chen X and Cong X: Apoptosis of mesenchymal stem cells induced by hydrogen peroxide concerns both endoplasmic reticulum stress and mitochondrial death pathway through regulation of caspases, p38 and JNK. J Cell Biochem 111: 967-978, 2010. 
5. Li Q, Wang Y and Deng Z: Pre-conditioned mesenchymal stem cells: A better way for cell-based therapy. Stem Cell Res Ther 4: 63, 2013.

6. Lee KA, Shim W, Paik MJ, Lee SC, Shin JY, Ahn YH, Park K, Kim JH, Choi S and Lee G: Analysis of changes in the viability and gene expression profiles of human mesenchymal stromal cells over time. Cytotherapy 11: 688-697, 2009.

7. Xu J, Qian J, Xie X, Lin L, Zou Y, Fu M, Huang Z, Zhang G, $\mathrm{Su}$ Y and Ge J: High density lipoprotein protects mesenchymal stem cells from oxidative stress-induced apoptosis via activation of the PI3K/Akt pathway and suppression of reactive oxygen species. Int J Mol Sci 13: 17104-17120, 2012.

8. Jeong DM, Jung HA and Choi JS: Comparative antioxidant activity and HPLC profiles of some selected Korean thistles. Arch Pharm Res 31: 28-33, 2008.

9. Yoo YM, Nam JH, Kim MY, Choi J and Park HJ: Pectolinarin and pectolinarigenin of Cirsium setidens prevent the hepatic injury in rats caused by d-galactosamine via an antioxidant mechanism. Biol Pharm Bull 31: 760-764, 2008.

10. Noh H, Lee H, Kim E, Mu L, Rhee YK, Cho CW and Chung J: Inhibitory effect of a Cirsium setidens extract on hepatic fat accumulation in mice fed a high-fat diet via the induction of fatty acid $\beta$-oxidation. Biosci Biotechnol Biochem 77: 1424-1429, 2013.

11. Thao NT, Cuong TD, Hung TM, Lee JH, Na M, Son JK, Jung HJ, Fang Z, Woo MH, Choi JS and Min BS: Simultaneous determination of bioactive flavonoids in some selected Korean thistles by high-performance liquid chromatography. Arch Pharm Res 34: 455-461, 2011.

12. Russo V, Young S, Hamilton A, Amsden BG and Flynn LE: Mesenchymal stem cell delivery strategies to promote cardiac regeneration following ischemic injury. Biomaterials 35: 3956-3974, 2014

13. Gimble JM, Katz AJ and Bunnell BA: Adipose-derived stem cells for regenerative medicine. Circ Res 100: 1249-1260, 2007.

14. Lee WB, Kwon HC, Cho OR, Lee KC, Choi SU, Baek NI and Lee KR: Phytochemical constituents of Cirsium setidens Nakai and their cytotoxicity against human cancer cell lines. Arch Pharm Res 25: 628-635, 2002.
15. Lee SH, Heo SI, Li L, Lee MJ and Wang MH: Antioxidant and hepatoprotective activities of Cirsium setidens Nakai against CCl4-induced liver damage. Am J Chin Med 36: 107-114, 2008.

16. Ahn MJ, Hur SJ, Kim EH, Lee SH, Shin JS, Kim MK, Uchizono JA, Whang WK and Kim DS: Scopoletin from Cirsium setidens increases melanin synthesis via CREB phosphorylation in B16F10 cells. Korean J Physiol Pharmacol 18: 307-311, 2014.

17. Lim H, Son KH, Chang HW, Bae K, Kang SS and Kim HP Anti-inflammatory activity of pectolinarigenin and pectolinarin isolated from Cirsium chanroenicum. Biol Pharm Bull 31: 2063-2067, 2008.

18. Matsuzawa A and Ichijo H: Redox control of cell fate by MAP kinase: Physiological roles of ASK1-MAP kinase pathway in stress signaling. Biochim Biophys Acta 1780: 1325-1336, 2008.

19. Cuevas BD, Abell AN and Johnson GL: Role of mitogen-activated protein kinase kinase kinases in signal integration. Oncogene 26: 3159-3171, 2007.

20. Chung YM, Park SH, Tsai WB, Wang SY, Ikeda MA, Berek JS Chen DJ and Hu MC: FOXO3 signalling links ATM to the p53 apoptotic pathway following DNA damage. Nat Commun 3: 1000, 2012 .

21. Lavin MF, Gueven N, Bottle S and Gatti RA: Current and potential therapeutic strategies for the treatment of ataxia-telangiectasia. Br Med Bull 81-82: 129-147, 2007.

22. Guo Z, Kozlov S, Lavin MF, Person MD and Paull TT: ATM activation by oxidative stress. Science 330: 517-521, 2010.

23. Chen BP, Li M and Asaithamby A: New insights into the roles of ATM and DNA-PKcs in the cellular response to oxidative stress. Cancer Lett 327: 103-110, 2012.

24. Mollazadeh S, Fazly Bazzaz BS and Kerachian MA: Role of apoptosis in pathogenesis and treatment of bone-related diseases. J Orthop Surg Res 10: 15, 2015.

25. Elmore S: Apoptosis: A review of programmed cell death. Toxicol Pathol 35: 495-516, 2007.

26. Virag L, Robaszkiewicz A, Rodriguez-Vargas JM and Oliver FJ: Poly(ADP-ribose) signaling in cell death. Mol Aspects Med 34: 1153-1167, 2013. 